\title{
Erratum to: Central European Journal of Geosciences, Volume 3, Number 2
}

\footnotetext{
Abstract: The papers in Volume 3, Number 2 (June 2011) were initially published on-line with the erroneous number 1 of volume 3 in the article pdf-files. The correct citation data for all the papers is listed below. The editorial staff of the Central European Journal of Geosciences apologize for any inconvenience that may result from the oversight.

(C) Versita Sp. z o.o.
}

1. János Kovács, Szabolcs Á. Fábián, Gábor Varga: Geomorphology in a changing environment. Cent. Eur. J. Geosci., 3(2), 100-101, DOI: 10.2478/s13533-011-0021-7

2. Karoly Németh, Corina Risso, Francisco Nullo, Gabor Kereszturi: The role of collapsing and cone rafting on eruption style changes and final cone morphology: Los Morados scoria cone, Mendoza, Argentina. Cent. Eur. J. Geosci., 3(2), 102-118, DOI: 10.2478/s13533-011-0008-4

3. Titusz Bugya, Szabolcs Á. Fábián, Noémi L. Görcs, István P. Kovács, Bertalan Radvánszky: Surface changes on a landslide affected high bluff in Dunaszekcsó (Hungary), Cent. Eur. J. Geosci., 3(2), 119-128, DOI: 10.2478/s13533-011-0014-6

4. Gabriella Barta: Secondary carbonates in loesspaleosoil sequences: a general review, Cent. Eur. J. Geosci., 3(2), 129-146, DOI: 10.2478/s13533-0110013-7

5. Rudolf Musil: Gravettian environmental changes in a $\mathrm{N}$ - S transect of Central Europe, Cent. Eur. J. Geosci., 3(2), 147-154, DOI: 10.2478/s13533-0110015-5

6. Alice Ghiselli, Marzio Merazzi, Andrea Strini, Roberto Margutti, Michele Mercuriali: Hypogeal geological survey in the "Grotta del Re Tiberio" natural cave (Apennines, Italy): a valid tool for reconstructing the structural setting, Cent. Eur. J.
Geosci., 3(2), 155-168, DOI: 10.2478/s13533-0110012-8

7. Rauf Gardashov: The distribution of sun glints sizes on the sea surface, Cent. Eur. J. Geosci., 3(2), 169174, DOI: 10.2478/s13533-011-0016-4

8. Daria Gushchina, Boris Dewitte: The relationship between intraseasonal tropical variability and ENSO and its modulation at seasonal to decadal timescales, Cent. Eur. J. Geosci., 3(2), 175-196, DOI: 10.2478/s13533-011-0017-3

9. Martin Michálek, Marián Putiš, Christoph A. Hauzenberger: P-T data of $\mathrm{Ky}$-St-Grt gneisses hosting HP amphibolites and eclogites from the Austroalpine Polinik complex, Kreuzeck Mountains, Eastern Alps, Austria, Cent. Eur. J. Geosci., 3(2), 197-206, DOI: 10.2478/s13533-011-0018-2

10. Jindřich Šancer, Martin Štrejbar, Aneta Maleňáková: Effects of cyclic loading on the rheological properties of sandstones, Cent. Eur. J. Geosci., 3(2), 207-214, DOI: 10.2478/s13533-0110020-8

11. George Migiros, George D. Bathrellos, Hariklia D. Skilodimou, Theodoros Karamousalis: Pinios (Peneus) river (Central Greece): hydrological - geomorphological elements and changes during the Quaternary, Cent. Eur. J. Geosci., 3(2), 215-228, 10.2478/s13533-011-0019-1 